Session 3120

\title{
Distributed Real and Virtual Learning Environment for Mechatronics
}

\author{
F. Wilhelm Bruns, Heinz-Hermann Erbe \\ ARTEC Center for Work, Environment, Technology \\ Bremen University/ \\ Institute of Professional Education \\ Technische Universität Berlin
}

\begin{abstract}
:
A European project DERIVE developed a learning environment where on-site and remote components merge into a cooperative learning process. The envisaged system allows to work together with complex real and virtual systems, consisting of parts which may be distributed remotely. The learning environment includes a supportive web-database with multimedia learning sequences providing theoretical background information, exercises and help to handle training tasks. Hardware equipment can be connected to the virtual environment with a special bi-directional sensor-actor coupling (hyper bond). The DERIVE learning environment smoothly integrates equipment and supports full hardware-in-the-loop functionality, allowing to build up real mechatronic systems as subsystems of complex virtual systems.
\end{abstract}

Keywords: bond graphs, hyper bonds, e-learning, simulation, remote experiments

\section{INTRODUCTION}

Engineering education are confronted with the need to develop theoretical integrated with practical learning sequences to fulfill the demands for multi-skilled engineers and also skilled technicians. Tasks and problem solving in mechatronics requires cognitive and operational knowledge and practical experience about building systems, diagnosis- and maintenancetechniques. However, a significant challenge is that these tasks are essentially characterized by the use of tele-medial systems. Service staff in the professional field need the ability to achieve their aims in (tele) cooperation with others, and they should be able to cooperate in virtual and supranational forms of organization. Concepts concerning pedagogical, technical and organizational aspects to meet these requirements in education and training are in development. Cultural differences and similarities concerning learning and collaboration styles have also to be considered regarding curricula, courseware and teaching methods.

Computers are now used in the classroom as multimedia tools to provide alternative sources of learning material, to provide interactive learning situations and to provide simulation of systems that cannot for reasons of cost, size or safety be used in reality. The use of the Internet is rapidly increasing and is being seen by some people as the greatest source of knowledge available for learning. The use of simulation tools has a number of benefits to education. The learner is not exposed to the hazards of the real world. The learner is able to explore a range of possible solutions easily and quickly. The learner is able to use the tools that will be available in industry.

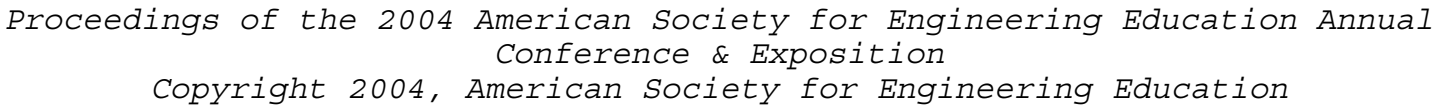


The cost of simulation tools is significantly less than the real world components and allows more participation and interaction than a limited demonstration. An added benefit is that learners today, enjoy using computer based technology and this enthusiasm fosters the learning process. The question is indeed to what extend real experience can be replaced by learning with simulations? The internet makes it possible for e-learners to have access to remote laboratories. They could change control parameters to study the effect on the performance of a plant equipment. Before doing this they can experiment with the virtual equipment in a computersimulation to save time when remote experimenting with the real equipment. However, reality and virtuality are separated. It could be of advantage to bridge the gap between reality and virtuality to a "mixed reality" by free switching between both in a bi-directional manner. To make this possible, Bruns ${ }^{1}$ developed a concept of Hyper-Bonds based on the meanwhile well established bond graph theory.

\section{BOND GRAPHS AND HYPER BONDS}

Hyper-Bonds combine the unified abstract systems representation of bond graphs with their real implementation in a "hyper-connection" between physical phenomena of the computer-external environment and the logical structure of computer-internal representations, a blend of physical systems with their virtual counterparts.

Paynter ${ }^{2}$ introduced the theory of bond graphs as a unifying view on physical phenomena from a continuity of power-flow perspective. Power flows through system components and connections by way that the product of effort and flow is continuous, following typical laws of energy conservation and power-flow continuity. Effort (e) is the driving force for flow (f) and can be a
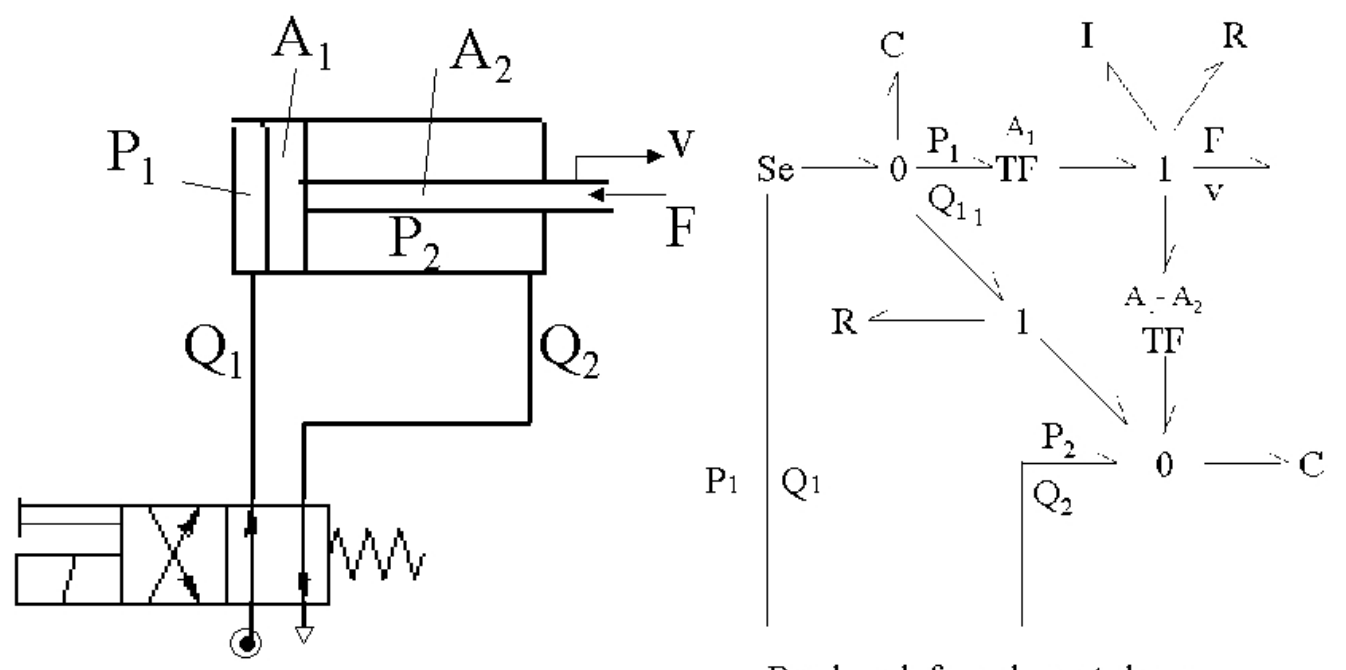

Bondgraph for valve not shown

Fig 1. 4/2 way valve controlling a double-acting cylinder and its bond graph

pressure difference, force and torque, electrical potential difference, temperature difference etc. Flow (f) can be a flow of material, momentum, electric current, entropy. The bond graph theory has been further developed by Karnopp ${ }^{3}$ et al (1990). Pairs of effort and flow (e,f) are for

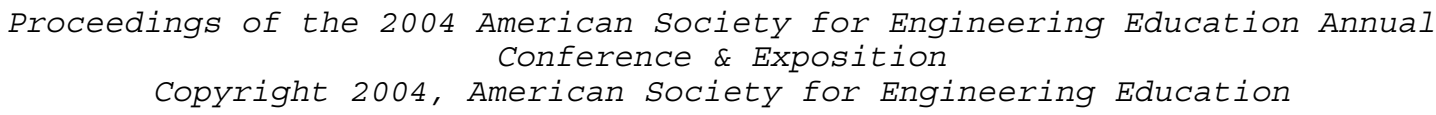


example in mechanical systems force (F) and velocity (v), in electrical systems voltage (V) and current (i), in pneumatic/hydraulic systems pressure $(\mathrm{P})$ and volume flow rate $(\mathrm{dQ} / \mathrm{dt})$. Figure 1 explains the correlation of pressure $\mathrm{p}$, fluid flow $\mathrm{q}$ with force $\mathrm{F}$, velocity $\mathrm{v}$ and mass/inertia $\mathrm{I}$, compressibility $\mathrm{C}$ of the fluid and friction $\mathrm{R}$ in a simple pneumatic equipment.

Components (valves, cylinders, etc.) are always connected by bonds having the value pair e and $f$ where one of them can be seen as input the other as output from a cause-effect point of view. However, which one is input and which one output can only be determined from an overall systems view by causal reasoning. Knowing e and $\mathrm{f}$ at one connection, resulting from calculation or measurement, allows a cutting of the system in two parts and a separate investigation.

In mechanics one learns the principle of cutting a system at well defined boundaries and replacing the external influences by some observable and measurable relevant variables, reducing the investigation to the internal dynamics of the rest. In laboratory work this principle is used to construct reproducible experiments, but also mentally it is used to think about systems in hypothesis and mental experiments. Today laboratories, being more and more penetrated by computers, a free and easy distribution of a system between reality and virtuality has some advantage. Certain well known aspects of a system can be represented in a formal way by algorithms in the computer, others to be investigated in more detail are represented in reality, but coupled to a dynamic surrounding. This allows completely new forms of easy experimental work and learning. Here hyper bonds come into play.

In order to provide arbitrary boundary conditions, we must have a mechanism to switch between source of effort and sink of effort, and to generate one phenomenon and sense the other. Figures 2 and 3 explain how the connection between reality and virtuality (and vice versa) is realized

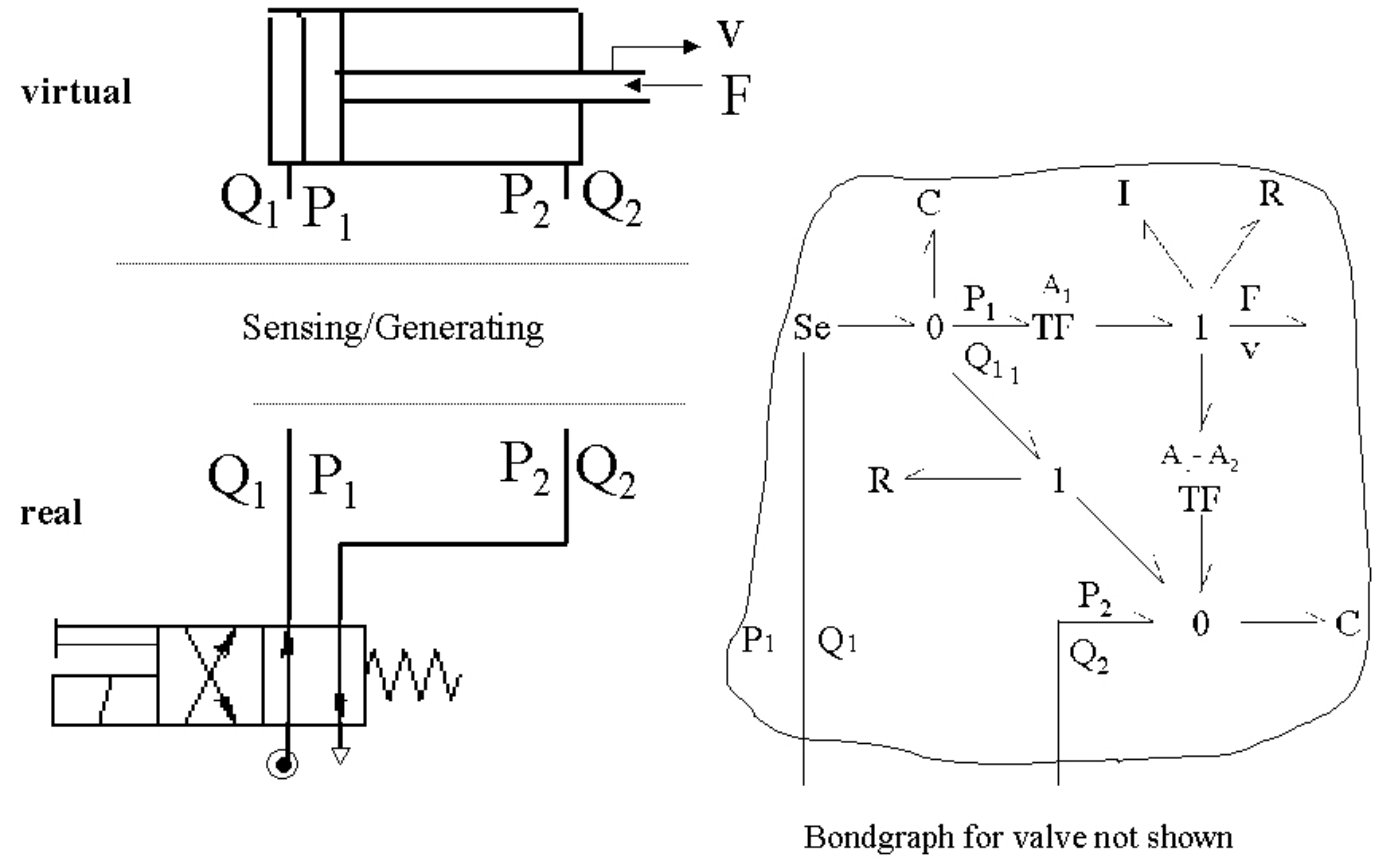

Fig. 2. Hyper-Bond for the system of Figure 1 maintaining mixed reality

with a special sensor/actuator coupling. Hyper bonds are of course also possible for pairs like voltage $\mathrm{V}$ and current $\mathrm{i}$, Temperature $\mathrm{T}$ and heatflow $\mathrm{dQ} / \mathrm{dt}$ and mass $\mathrm{M}$ and velocity $\mathrm{v}$.

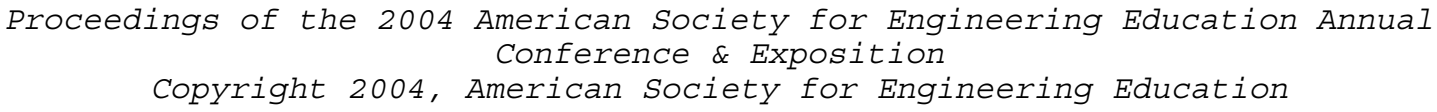


Hyper bond is a mechanism based on the translation between physical effort/flow phenomena and digital information like any other analog/digital and digital/analog conversion, however it aims at a unified application oriented solution connecting the physical and its virtual representation and continuation. Figures 2 and 3 are highly simplified presentations. For a deeper understanding of hybrid bond graphs and how to handle discontinuities, boundary cuts and transfer between power flow, signal flow and logic switches see Mostermann ${ }^{4}$.

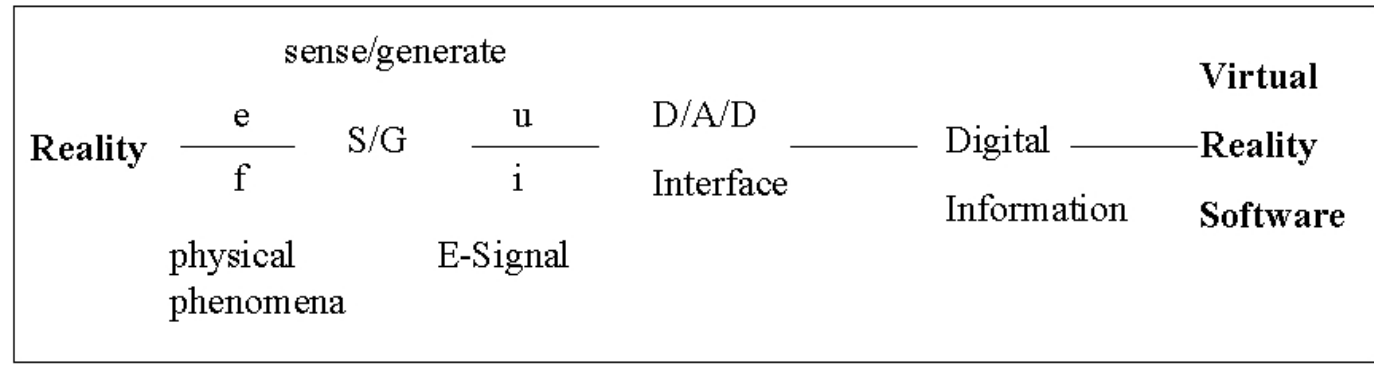

Fig. 3. Hyper Bond

\section{LEARNING ENVIRONMENTS}

The European project "Distributed Real and Virtual Learning Environment for Mechatronics and Tele-service" (DERIVE) used the concept of hyper bonds to describe electro-pneumatics (Bruns ${ }^{5}$ et al) in a unified and didactically expandable way as well as to have a link to several powerful simulation tools supporting bond graph modeling.

DERIVE provides a new learning environment that supports schools for technicians to deliver courses in mechatronics. The support for the learning process will be reflected in a graduation from local real to local virtual to remote virtual to remote real, taking the student from basic knowledge to the full implementation in industry.

The tele-cooperation functionality in the learning environment will allow enterprises to use the training facilities of vocational schools and/or other providers for updating their employees. With new equipment being more complex and requiring more complex maintenance, the training requirements for workforce and engineers increases. The new environment will allow groups of employees at remote locations to take part at the same training using the same equipment (either simulated or real ). This staff will be able to work in a collaborative way to solve problems and explore learning situations. This new kind of interaction will allow the systematic support of skilled workers and engineers. Also, the DERIVE learning environment is an appropriate tool to realize project orientation in technical training, providing a platform for self-managed and collaborative learning.

The project developed a mixed reality human computer interfaces (Figure 4). To develop adequate technological and pedagogical concepts for e-learning in future technical training, user need must be analyzed in depth. The requirements of different user groups (students, teachers, employees) were described and consolidated. Acting in an environment, where real world objects and IT-technologies are applied simultaneously, requires new concepts of supporting cooperating local and distributed learning groups. The scientific challenge is to handle physical as well as virtual presence and awareness without confusing side effects for the users. DERIVE evaluated the user-friendliness of the developed software, analyzed the established

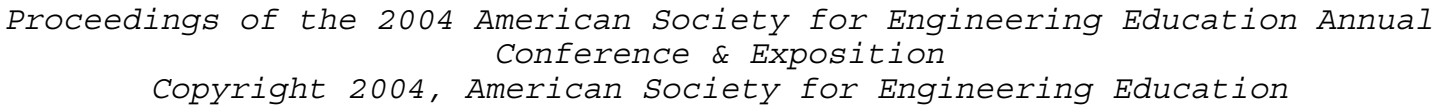


communication and tele-cooperation behavior and qualified the learning benefit of the system prototype.

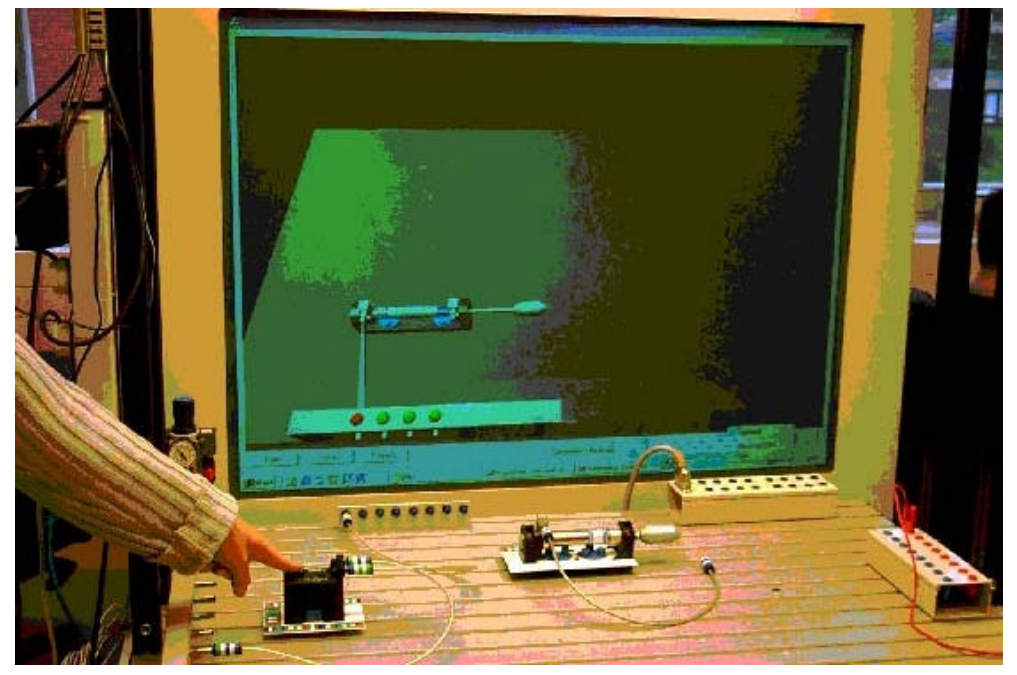

Fig. 4. Pneumatic equipment similar to figures 1 and 2 split in reality and virtuality (single acting cylinder)

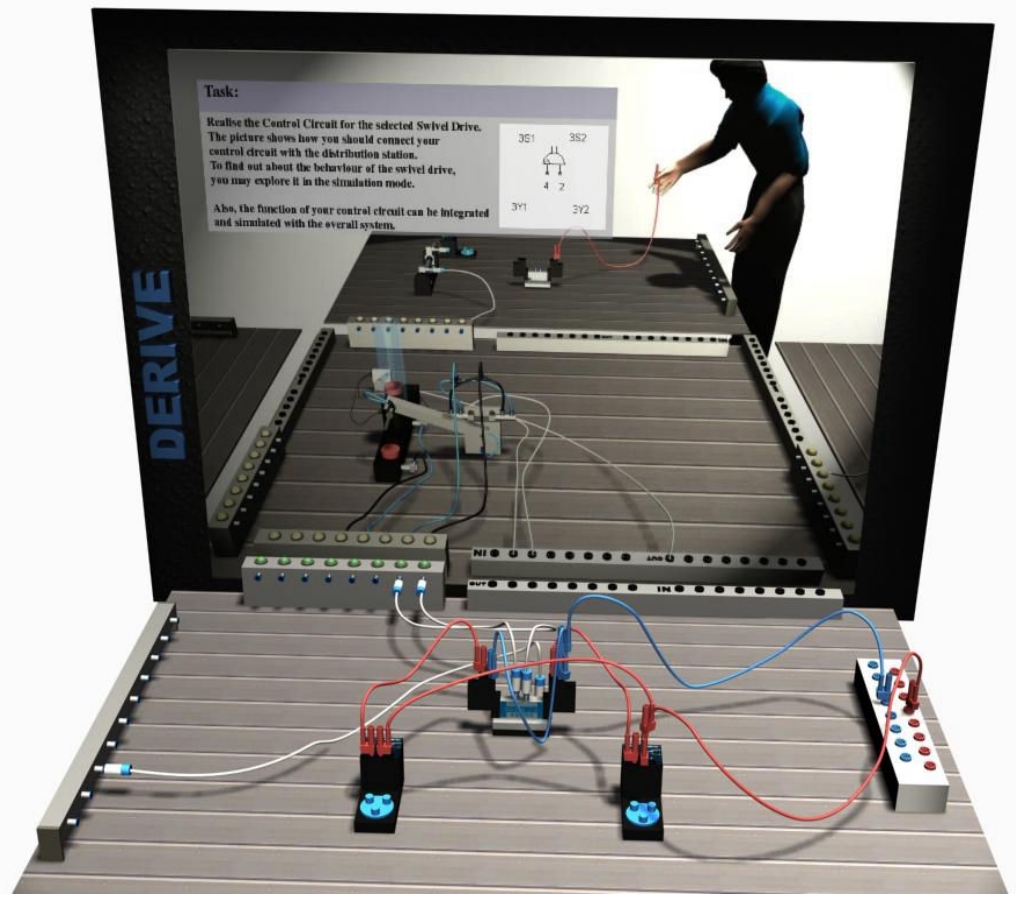

Fig. 5. Real and virtual environment for training in electro-pneumatics to control a robotarm

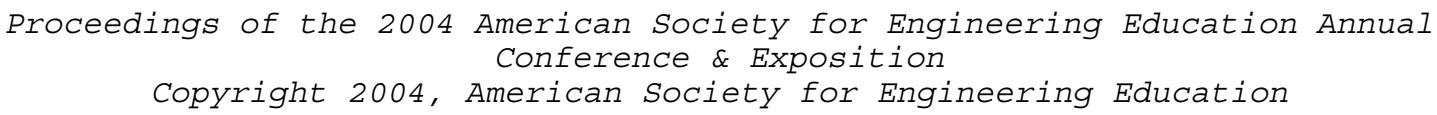


DERIVE developed a mechatronic learning environment where on-site and remote components merge into a cooperative learning process. The envisaged system allows to work together with complex real and virtual mechatronic systems, consisting of parts which may be distributed remotely. The learning environment includes a supportive web-database with multimedia learning sequences providing theoretical background information, exercises and help to handle training tasks.

The DERIVE learning environment smoothly integrates equipment and supports hardware-inthe-loop functionality, allowing to build up real mechatronic systems as subsystems of complex virtual systems (Figure 5).

The environment has been evaluated in 3 European vocational schools and colleges and at one industrial site. The results, based on a prototype which was not yet very stable, was already very encouraging for further offering a broad learning-landscape to navigate between concrete and symbolic representations. In a comparative evaluation of

- traditional teaching with blackboard and teacher learner interaction

- support by simulation only (FluidSim-software of FESTO AG, Germany)

- support by real complex system only (Modular Production System/MPS of FESTO AG, Germany)

- $\quad$ support by real and virtual media (DERIVE)

in 20 hours-courses of mechatronics, Grund and Grote ${ }^{6}$ found some hints that students/apprentices being trained with DERIVE showed better performances in construction tasks and fault finding, whereas for the factual knowledge no significant differences could be observed.

\section{DIDACTICAL HINTS}

As the profession of mechatronics is rather new, there is no elaborated didactic for mechatronics education yet. The following main tendencies in mechatronics are considered important:

- thinking in abstract categories of structure-behavior-function, searching for alternative concrete instantiations (e.g. various structures for one function, or various functions of one structure)

- thinking in categories of information-control-work process and their realization

- searching for unified views of systems dynamics (e.g. Petri-Nets and Bond Graphs) for analogous physical phenomena (electricity, pneumatics, force-momentum mechanics ...)

- judging about the adequateness or failure of simulation models versus real systems

A theoretical framework for this didactic requires more insight into how individual learning styles use individual learning media and paths to develop meaning and concepts from basic experiences with natural and technical phenomena. However the development of learning environments should not wait on such a foundation. Instead, we suggest some iteration between Learning Environment development and theoretical derivations based on empirical studies. In several research projects, we found hints for further extensions of learning theory and practice.

Tasks and problem solving in mechatronics requires cognitive and operational knowledge and practical experience about building systems, diagnosis- and maintenance-techniques. However, a significant challenge is that these tasks are essentially characterized by the use of tele-medial

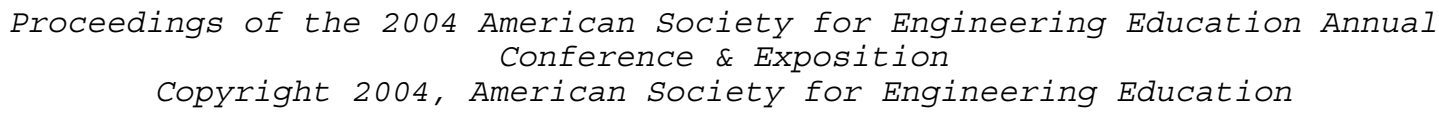


systems. Service staff in the professional field need the ability to achieve their aims in (tele) cooperation with others, and they should be able to cooperate in virtual and supranational forms of organization. Until now there exist no elaborated concepts concerning pedagogical, technical and organizational aspects to meet these requirements in education and training. Cultural differences and similarities concerning learning and collaboration styles can be noticed but have not sufficiently been integrated into curricula, courseware and teaching methods.

Enterprises have come to realize that employee expertise is a vital and dynamic living treasure. The desire for employee expertise is meaningless unless an organization (enterprise) can develop it in ways that respond to its business needs. Many enterprises rely on off-the-job training (formal learning) without considering its suitability for the learning tasks at hand. On-the-job training (informal learning) has a substantial advantage: it is more close to the problems to be solved and it can be organized in a cooperative way crossing the border between different professions that are involved in a project to fulfill an order of a customer. But on-the-job training is often unplanned and therefore mostly ineffective. With a structure and using well prepared training material usable at the workplace and facilitating advises from outside for cooperative learning makes on-the-job training a powerful tool, Jacobs and Jones ${ }^{7}$ :

- employees work in projects (small groups of different professions) to solve problems,

- employees learn how to learn and think critically,

- employees identify the skills needed to meet the requirements posed by current work,

- employees develop a personal theory of management, leadership or empowerment.

What enterprises need is a culture of expertise, for the good of both the enterprise and the individuals in it. Employees should be encouraged to engage in continuous learning activities but not to forget that learning and doing go hand in hand. Learning by itself does not lead to enhanced productivity. But expertise can be attained only through learning. Having a learning culture is prerequisite to promote expertise. Analyzing a task to be learned often brings insights into ways of performing a task. Training modules can be used as a way of documenting and storing task information for purposes other than training.

Collecting case studies of solved problems in manufacturing and service could help to solve new tasks. If they are released and are available in electronic format, preferably via internet, it is a powerful instrument for learning at workplaces. Via the internet it is also possible to use training material and remote labs offered by training institutions.

\section{CONCLUSIONS}

Universities and Technical Training Schools are challenged to provide the industry with engineers and skilled workers for the interdisciplinary field of Mechatronics. As this field of research and application is in a steady development the curricula cannot be fixed for a long period as it is mostly the case in mechanical and electrical engineering education courses. Education and training institutions have to be in close co-operation with industry demands, and to transfer research results into praxis via educating the personnel.

\section{BIBLIOGRAPHIC INFORMATION}

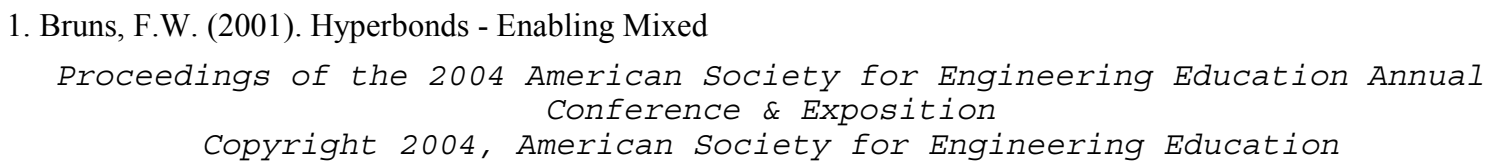


Reality. artec paper 82, Bremen

ftp://artec-nt.artec.uni-bremen.de /pub/Field1/Publications/

artec-01-Bruns-hyperbonds.pdf

2. Paynter, H. M. (1961). Analysis and Design of Engineering Systems, MIT Press, Cambridge, MA.

3. Karnopp, D. C., Margolis, D. L., Rosenberg, R. C.(1990). System Dynamics - A unified Approach John Wiley, New York.

4. Mostermann, P. J. (1997). Hybrid dynamic systems: A hybrid Bond Graph Modeling Paradigm and its Application in Diagnosis. Dissertation, Vanderbilt University, Nashiville, Tennessee

5. Bruns, F.W. et al (2002). DERIVE, Final Report. artec-paper 102, Bremen ftp://artec-nt.artec.uni-bremen.de /pub/Field1/Publications/ artec-02-Bruns-Final_Report_Derive.pdf

Grund, S., Grote, G. (2004): Specific Learning Effects of a Mixed Reality System in Automation Technology Training. In print

7. Jacobs, R.L. and M.J. Jones (1995). Structured Onthe-Job Training. Berrett-Koehler Publisher, San Francisco.

\section{BIOGRAPHIC INFORMATION}

Friedrich-Wilhelm Bruns, Prof. Dr.-Ing., born 23. 5. 1945

1983 - 1987 Founder and Director of a Software-Company for CA-Design and Numerical Control

1987 - present Professor of Mechanical Engineering, Professional Education and Work-Design at University of Bremen

Heinz-Hermann Erbe, Prof. Dr.-Ing., born 15.03.1937

1980 - 1986 Professor of Mechanical Engineering and Professional Education, University of Bremen 1986 - Present Professor at the Institute of Professional Education ,Technical University Berlin Chair of the IFAC-Technical Committee on Cost Oriented Automation (1999 - 2005),

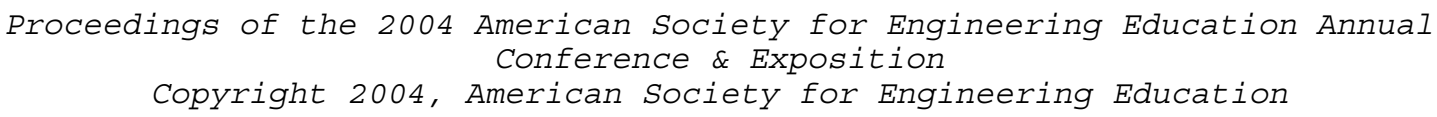

